\title{
SKED program for a modified Sternberg procedure
}

\author{
ROBERT D. DINGLER, E. H. UHLENHUTH, and MARTIN STERN \\ University of Chicago, Pritzker School of Medicine, 950 East 59th Street, Chicago, Illinois 60637
}

\begin{abstract}
For this procedure, a number of machine language subroutines have been integrated within the SKED system. This provides a flexible system for controlling sequential events in studies of pharmacological effects on performance of psychomotor tasks by human subjects. A description of the procedure and examples of each subroutine's use will be presented.
\end{abstract}

As a process control language developed for use in the behavioral research laboratory, SKED (Snapper, Knapp, \& Kushner, 1970) has provided the researcher with a valuable tool for experimental control and data recording. Even though SKED has proven its usefulness in a wide variety of applications, some users will inevitably find that they require some functions that are not of sufficiently general use to have been included in the original system. It is for this reason that a method has been developed in which the run time system (RTS) can pass program control to special purpose subroutines, written by the experienced user.

Each special purpose subroutine can be assembled with PAL8, inserted in unused portions of any field, and protected from being overlaid by the state tables. When necessary, the subroutine can then be called from the state table by means of an F3 statement. The format for this statement is F3 (Oxxxx, Argl...ArgN) where Oxxxx is the octal address of the subroutine and Argl...ArgN are optional arguments used to set parameters of the subroutine when necessary.

\section{SYSTEMS DESCRIPTION}

Because this particular procedure and the F3s described below have been developed for a particular installation, a description of this system is necessary.

The basic system is composed of a PDP8/E digital computer, operating under the $8 \mathrm{~K}$ Programming System, PS/8; a DK8-EP clock; a dual TCO8 Dectape controller and dual TU56 Dectape transports; a UDC8 interface; a Decwriter; and a VR-14 cathode ray tube (CRT). All of the above equipment is manufactured by Digital Equipment Corporation. A modification of the DECUS version of SKED provides for experimental control and data recording. Major additions to this version of SKED include: (1) an initial dialogue that asks the operator for daily constants and program names; (2) Dectape handling routines; and (3) a number of F3s. In addition, a modification has been made to the compiler so that all SKED commands that can access core locations in

This work was supported in part by FDA Grant No. 72-42 (E. H. Uhlenhuth, Principal Investigator).

Martin Stern is now at G. D. Searle and Company, Skokie. Illinois. memory, can also access the recording counters. Thus, any numeric value in the state table prefaced by a " + " refers to the core location of that recording counter.

\section{PROCEDURAL DESCRIPTION}

The procedure of interest here is a modificationof a procedure developed by Sternberg (1966), in which the latency of a response is measured under various drug and reinforcement conditions. At the start of a session, the word "Ready" is projected on the CRT. After the subject has placed an index finger on each of two response buttons located in front of him, a series of digits ranging in length from one to six are displayed on the CRT sequentially for $1.2 \mathrm{sec}$ each. Three seconds after the last digit has been displayed, a single test digit appears, accompanied by a tone. The subject then lifts his right index finger to indicate that the test digit was a member of the preceding series or his left index finger to indicate that it was not. The test stimuli remain on until terminated by a correct response.

Each session consists of seven sets of 12 trials each. The stimulus sets are designed so that each series length occurs twice in random order within each set of trials, once with a test digit in the series (positive trial) and once with a test digit not in the series (negative trial). After every set of trials, a score in dollars and cents based on the speed and accuracy of the subject's performance, is displayed on the CRT. Twelve correct responses averaging the preselected latency (determined by the initial dialogue) produce a display of $\$ 2.00$. For each error, $\$ .50$ is deducted. For each correct response, $\$ .01$ is added for every $10-\mathrm{msec}$ improvement below the preselected latency criterion and $\$ .01$ is deducted for every $10-\mathrm{msec}$ delay above the preselected latency criterion.

Each session is divided into two parts, reinforced and nonreinforced. The first set of trials for each session is defined as a practice set, and latency measures are not included in computation of the average latency. Of the remaining six sets of trials, three sets constitute the reinforced part of the procedure and the subject is paid the actual amounts displayed on the CRT. The remaining three sets are the nonreinforced part and the subject is paid a base pay, regardless of his actual 
performance. The latency measures from the reinforced and nonreinforced parts are then combined, and an average latency is computed. This average is then adjusted so that each subject will normally earn about $\$ 6$ per session.

\section{DESCRIPTION OF THE F3S}

Concurrent with the development of this procedure, a number of F3s have been incorporated into the RTS. Specifically, F3s have been developed that (1) open input files and read data from these files; (2) do various plotting functions for the CRT; and (3) calculate winnings that are then displayed on the CRT.

Since all of the subroutines are located in Field 1, all F3s first branch to a common location in Field 0. The general format for each F3 is F3 $(03100, \mathrm{~N}$, Argl,...ArgN), where 03100 is the octal entry point in Field 0 for all F3s, $N$ is the specific type of F3, and Argl...ArgN set the parameters for each F3. In the following description of the F3s, A, B, C, etc., will stand for a memory address, and (A), (B), (C), etc., will stand for the contents of a memory address.

\section{Examples of the F3s}

The following is a list of the general form of each F3 used in the modified Sternberg procedure:

(1) F3 $(03100,1,1)$

(2) F3 $(03100,2, A,(B))$

(3) F3 $(03100,3, A,(B), X, Y, S)$

(4) F3 $(03100,4)$

(5) F3 $(03100,5, \mathrm{~A},(\mathrm{~B}))$

(6) F3 $(03100,6,3, A,(B), C,(D))$

In the following description of the use of each F3, F3(1) will describe the first F3 of the above list, F3(2) the second F3, etc.

At the start of each session, F3(1) opens an input file that was previously generated by a Focal program; then, prior to each trial, data for that trial is read by F3(2). Functionally, this $F 3$ reads the next digit from the input file and stores it at Location A + (B).

When a character is to be displayed on the CRT, the state table will call either F3(3) if it is the only character or the first character in a string of characters, or F3(5) if at least one other character has previously been displayed. F3(3) will display on the CRT the character whose number is stored in Location $\mathrm{A}+(\mathrm{B})$ at Coordinates $X, Y$ with Size $S$. If the call is for F3(5), the character whose number is stored in Location $A+(B)$ will be displayed at the immediate right of the previously displayed character. When the display is to be terminated, F3(4) will then clear the display screen.

It should be noted that the VR-14 is not a storage scope. This means that the characters that are displayed must be stored in core memory. At present, each character requires four memory locations to describe it within a 5 by 8 dot matrix that is projected on the CRT. What this means is that it becomes expensive in terms of computer memory if it is necesary to display the complete set of alphanumeric characters.

The final F3 mentioned, F3(6), is used to calculate the winnings or losings of a set of 12 trials. The number 3 in the F3 list indicates that this call is for the Stemberg experiment. In effect, this F3 takes the content of Location A + (B) (the preselected base minus the latency of the response), multiples this result by the contents of (D) (equal to a multiplier typed in during the initial dialogue), and stores the result in Location C.

\section{Systems Development}

Our major objective in the development of the previously mentioned F3s, is to expand the ability of SKED to communicate with subjects in an experiment. The present system is a step on the way toward developments where experimental subjects will interact with the program entirely by means of highly versatile standard terminals, such as a CRT terminal. It will then be possible to orderly control a large variety of even more complex experiments.

\section{REFERENCES}

Snapper, A. G., Knapp, J. Z., \& Kushner, H. K. Mathematical description of schedules of reinforcement. In $W . N$. Schoenfeld (Ed.). The theory of reinforcement schedules. New York: Appleton-Century-Crofts, 1970. Pp. 247-275.

Stemberg, $S$. High-speed scanning in human memory. Science, $1966,153,652-654$. 